\title{
Applying Interactive Mechanism to Virtual Experiment Environment on WWW with Experiment Action Language
}

\author{
Rita Kuo, Maiga Chang and Jia-Sheng Heh \\ Dept. of Information Computer and Engineering, \\ Chung-Yuan Christian Univ., Chung-Li, 320, Taiwan \\ rita@mcsl.ice.cycu.edu.tw,maiga@ms2.hinet.net, jsheh@ice.cycu.edu.tw
}

\begin{abstract}
To build an interactive mechanism for distance learning environment such as a scientific experiment, the architecture of learning environment and assisted agents should be taken into consideration first. Another issue for constructing interactive mechanism is protocols between the environment and agents. An Experiment Action Language is designed for this purpose. Besides, the way for agent inferring rules is also presented in this paper.

\section{Experiment Environment in WWW}

A disadvantage of distance learning on Web, the lack of interaction between user and computer, makes the teaching procedure inefficient. Hence, a learning environment, like Virtual Experiment Environment (V.E.E.), with interactive mechanism supported for scientific experiment on WWW is needed.[6][7]

Some distance learning systems now propose agents for managing system files [10], notifying grades in on-line quiz system [8], diagnosing problems in problem solving system [1][2][5], a recorder of the system [3][4], and so on. This paper will support an interactive mechanism for agents to let agents own the ability to guide and assist students by getting information from V.E.E.. Besides, the protocol used to communicate with both V.E.E. and agents will also be discussed.
\end{abstract}

\section{Architecture of Interactive V.E.E}

To build a proper environment for student experiment on web, there are four components in V.E.E. as shown in Figure 1. Accompany with an Interactive V.E.E., an agent called Experiment Agent is used to assist learner during experimenting. Figure 2 illustrates that there are three sorts of interactions among V.E.E., Experiment Agent, and User.

In order to have the assistant ability, the Experiment Agent should be able to infer. There are five components make the architecture of experiment agents completely presented in Figure 3. To make an Experiment Agent intelligent, Knowledge Base is a necessary component and makes the Inference Engine get facts more properly. The results reasoned by Inference Engine will be transmitted to the Knowledge Translator, and then will be translated to appropriate Experiment Action Language before sent out to V.E.E. through using Communication Device.

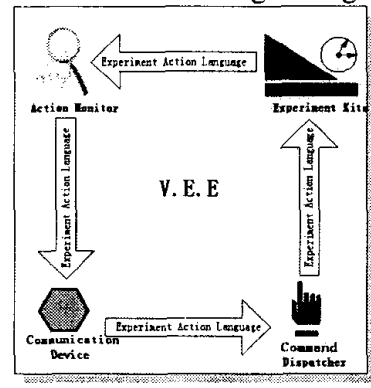

Figure 1

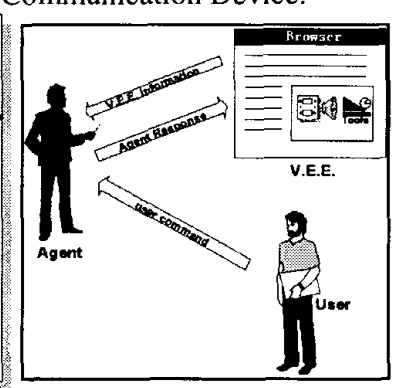

Figure 2.
Architecture of I.V.E.E. Interactions

Similarly, the Experiment Action Language transferred from V.E.E. will also be received by the Communication Device and then be sent to Knowledge Translator. Information Filter deletes the garbage information translated by the translator and store useful parts into Knowledge Base.

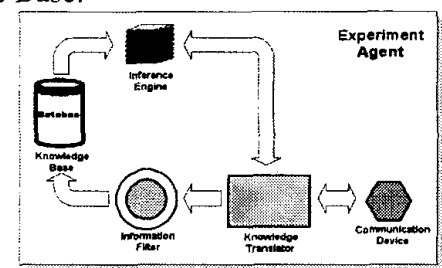

Figure 3. The Architecture of Experiment Agent

\section{Interactive Protocol Develop}

Experiment Action Language is a language for agent to control V.E.E. and record each step proceeded from learners. To let most of learners realize what command they call easier, scripting language is selected to be the language type.[9] The Experiment Action Language format lists as below:

command [object] [-option] [parameter] 
Inference Engine will divide the knowledge translated and pass from the Knowledge Translator into three conditions, including correct, inexactitude and error listed in Table 1.

\begin{tabular}{|c|c|c|c|c|}
\hline $\begin{array}{c}\text { Command } \\
\text { Condition }\end{array}$ & Command & object & option & parameter \\
\hline Correct & Correct & Correct & Correct & Correct \\
\hline \multirow{2}{*}{ Inexactitude } & Correct & Correct & Error & - \\
\cline { 2 - 5 } & Correct & Correct & - & Error \\
\hline \multirow{2}{*}{ Error } & Correct & Error & - & - \\
\cline { 2 - 5 } & Error & - & - & - \\
\hline
\end{tabular}

Table 1. The relationship between determined conditions and EAL (symbol, '-", means "don't care.")

To implement the interactive mechanism of V.E.E., Visual Lab is chosen for the experiment environment in this paper.[11] Taking a real action in Visual Lab for example, a learner moves the vertical ruler to the new position $(x l, y 1)$ could be written as move Ver_Ruler -u $x 1 y 1$ in EAL. This command asks agent to "move" the object "Ver_Ruler" (Vertical Ruler) to the new position " $x 1, y 1$, " and the option " $u$ " let agent know that $(x 1, y 1)$ is the position of the upper-left corner of Vertical Ruler.

By using the interactive mechanism analyzed and designed above, an Experiment Agent can either cheer students or provide suggestion when they doing correct or inexactitude experimental procedure as Figure 6 and Figure 7 shown.

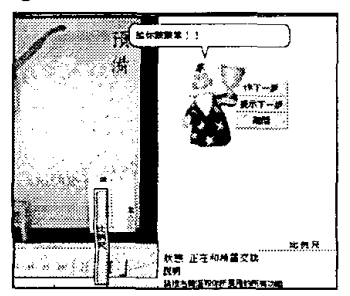

Figure 6

correct step in V.E.E.

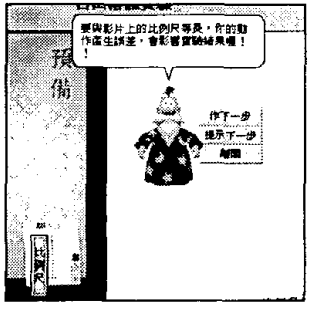

Figure 7.

inexactly step in V.E.E.

\section{Conclusion}

This paper proposes an interactive Virtual Experiment Environment and the architecture of Experiment Agent. Besides, an intercommunication protocol, Experiment Action Language, is also designed to realize the communication issue between V.E.E. and agents. However, since the interactions will also happen between agents, Knowledge Query Manipulate Language (KQML), is necessarily taken into consideration future. Beside the formation of rules and the intercommunicate protocol, the relations between Inference Engine and Information Filter might need further analysis for the automatic learning issue.

\section{References}

[1] Janie Chang, Maiga Chang and Jia-Sheng Heh (1999), "Applying the Evaluation Model of Problem Solving to Agent-based Instructional System :" Proceedings of the 7 th International Conference on Computers in Education, Chiba, Japan, 1999, pp. 141-148

[2] Janie Chang, Maiga Chang, Jeng-Lun Lin and JiaSheng Heh (2000), "Implements a Diagnostic Intelligent Agent for Problem Solving in Instructional Systems," Proceedings of the IEEE Int. Workshop on Advanced Learning Technologies, Palmerston North, New Zealand, pp.29-30, Dec. 4-6, 2000

[3] Chi-Wei Huang, Shiao-Ting Sun, Chia-Chin Chang, Kuo-Chang Jan and Kun-Yuan Yang (1998), "Education Software and Hardware", Proceedings of the 2nd Global Chinese Conference on Computer in Education, Hong Kong, 1998, pp.50-57

[4] Chi-Wei Huang, Chang-Kai Hsu, Maiga Chang and Jia-Sheng Heh (1998), "Designing an Open Architecture of Agent-based Virtual Experiment Environment on WWW," World Conference on Educational Multimedia, Hypermedia \& Telecommunications (ED-Media), 1999, Seattle, WA, USA, pp.270-275

[5] Jia-Sheng Heh (1999), "Evaluation Model of Problem Solving," Mathematical and Computer Modelling, Vol.30, 1999, pp.197-211

[6] Yu-Wei Jeng, Maiga Chang, Ivory Chung and JiaSheng Heh (1996), "Designing Objects for Virtual Experiments," OOTA'96, Taiwan, 1996, pp.331-339

[7] Li-Ping Kuo, Da-Xian Dong, Chang-Kai Hsu and JiaSheng Heh (2000), "Design an Enhanced Virtual Experiment Environment Using Science Process Skills on WWW," World Conference on Educational Multimedia, Hypermedia \& Telecommunications ( $E D$ Media), 2000. Montreal, Canada, Jun. 25- Jul. 1, 2000, pp. 1785

[8] A. Okada, H. Tarumi and Y. Kambayashi (2000), "Real-time quiz functions for dynamic group guidance in distance learning systems," Proceedings of the First International Conference on Web Information Systems Engineering, 2000, Vol.2, pp.188-195

[9] Robert W. Sebesta (1999), Concepts of Programming Languages, Addison-Wensley, 1999

[10] A. Shimano and H. Kuramae(2000), "Design and construction of educational computer system using self-maintenance system for files and user identification agent," Proceedings of the $9^{\text {th }}$ IEEE International Workshop on Robot and Human Interactive Communication, 2000. RO-MAN 2000, pp. 23-28

[11] Chao-Chiu Wang, Maiga Chang, Chang-Kai' Hsu and Jia-Sheng Heh (2000), "Visual Lab - A Multimedia Virtual Experiment Environment on WWW," World Conference on Educational Multimedia, Hypermedia \& Telecommunications (ED-Media), 2000, Montreal, Canada, Jun. 25- Jul. 1, 2000, pp.1509-1510 